The azimuthal component of Poynting's vector and the angular momentum of light

This content has been downloaded from IOPscience. Please scroll down to see the full text.

2015 J. Opt. 17125610

(http://iopscience.iop.org/2040-8986/17/12/125610)

View the table of contents for this issue, or go to the journal homepage for more

Download details:

IP Address: 130.209.115.82

This content was downloaded on $05 / 11 / 2015$ at $11: 13$

Please note that terms and conditions apply. 


\title{
The azimuthal component of Poynting's vector and the angular momentum of light
}

\author{
Robert P Cameron ${ }^{1}$, Fiona C Speirits ${ }^{1}$, Claire R Gilson ${ }^{2}$, L Allen ${ }^{3}$ and \\ Stephen M Barnett ${ }^{1}$
}

${ }^{1}$ School of Physics and Astronomy, University of Glasgow, Glasgow G12 8QQ, UK

${ }^{2}$ School of Mathematics and Statistics, University of Glasgow, Glasgow G12 8QQ, UK

${ }^{3}$ Department of Physics, University of Strathclyde, Glasgow G4 0NG, UK

E-mail: r.cameron.2@research.gla.ac.uk

Received 5 June 2015, revised 11 August 2015

Accepted for publication 12 August 2015

Published 29 October 2015

\begin{abstract}
The usual description in basic electromagnetic theory of the linear and angular momenta of light is centred upon the identification of Poynting's vector as the linear momentum density and its cross product with position, or azimuthal component, as the angular momentum density. This seemingly reasonable approach brings with it peculiarities, however, in particular with regards to the separation of angular momentum into orbital and spin contributions, which has sometimes been regarded as contrived. In the present paper, we observe that densities are not unique, which leads us to ask whether the usual description is, in fact, the most natural choice. To answer this, we adopt a fundamental rather than heuristic approach by first identifying appropriate symmetries of Maxwell's equations and subsequently applying Noether's theorem to obtain associated conservation laws. We do not arrive at the usual description. Rather, an equally acceptable one in which the relationship between linear and angular momenta is nevertheless more subtle and in which orbital and spin contributions emerge separately and with transparent forms.
\end{abstract}

Keywords: electrodynamics, OAM, Noether

\section{Introduction}

The idea is now established that a beam of light can possess well-defined angular momentum in the direction of propagation [1-3] and that this can be separated into an orbital contribution, attributable to helical phase fronts or the spatial distribution of the light [4], and a spin contribution, attributable to circular polarisation or the vectorial character of the electromagnetic field [5]. The explicit description in basic electromagnetic theory ${ }^{4}$ of the angular momentum of light

\footnotetext{
4 In the present paper, we consider light that is propagating freely within the classical domain. Thus, the electric and magnetic fields $\mathbf{E}$ and $\mathbf{B}$ which comprise the light obey Maxwell's equations as written in the strict absence of charge [6]. We work in a system of units in which the electric and magnetic constants $\epsilon_{0}$ and $\mu_{0}$ are equal to unity.
}

Content from this work may be used under the terms of the Creative Commons Attribution 3.0 licence. Any further distribution of this work must maintain attribution to the author(s) and the title of the work, journal citation and DOI. and in particular its separation into orbital and spin contributions remains somewhat poorly understood, however.

One usually starts by identifying Poynting's vector [7]

$$
\mathbf{g}=\mathbf{E} \times \mathbf{B}
$$

as the linear momentum density and

$$
\mathbf{j}=\mathbf{r} \times(\mathbf{E} \times \mathbf{B})
$$

as the angular momentum density, $\mathbf{j}$ being the cross product of position $\mathbf{r}$ with $\mathbf{g}$. The $z$ component $j_{z}$ of $\mathbf{j}$ can thus be thought of as the $\phi$ or azimuthal component of $\mathbf{g}$ as we shall elucidate in what follows. These identifications are justified in that $\mathbf{g}$ and $\mathbf{j}$, when integrated over all space ${ }^{5}$, yield the total linear and angular momenta, which are the generators of translations in space and rotations [6]. Moreover, $\mathbf{g}$ and $\mathbf{j}$ appear in continuity equations derivable from Maxwell's equations that embody the conservation of linear and angular momenta $[6,7]$. With the use of an integration by parts, the

${ }^{5}$ We assume where relevant that the light falls off suitably as $|\mathbf{r}| \rightarrow \infty$. 
total angular momentum can be separated into orbital and spin contributions, which are themselves separately conserved [8-10].

This approach seems reasonable but nevertheless brings with it peculiarities. For a single circularly polarized plane wave propagating in the $z$ direction [11]

$$
j_{z}=0
$$

suggesting that the wave possesses no spin in the direction of propagation, in apparent conflict with intuition [5] and indeed, experimental observation [12]. It can be argued that a single plane wave is unphysical, as it is of infinite extent. Yet curious results are also encountered when $\mathbf{j}$ is calculated for beams of light of finite spatial extent $[4,13,14]$ and it is clear, in fact, that $\mathbf{j}$ will never describe spin in a transparent manner, as $\mathbf{j}$ depends explicitly upon $\mathbf{r}$ whereas spin must be independent of the location of the origin. The orbital and spin contributions to the total angular momentum do exhibit transparent forms, but are often regarded as being not separately meaningful as their associated operators do not separately obey the usual angular momentum commutation relations $[15,16]$.

These peculiarities do not amount to any fundamental difficulties: careful treatments of light-matter interactions predict angular momentum transfers that do indeed match those observed in experiment $[11,13,17]$ and the orbital and spin contributions to the total angular momentum do indeed generate separately valid rotations, of the spatial distribution of the light [18] and of the orientations of the field vectors $[15,16,18]$. Nevertheless, the form taken by $\mathbf{j}$ remains puzzling and the separation of the total angular momentum into orbital and spin contributions has sometimes been regarded as contrived. One can ask, moreover, about the local and not just global separation of angular momentum into orbital and spin contributions.

The present paper was motivated by a desire to better understand these issues. We begin with the observations that (i) densities and indeed flux densities are not unique in yielding total quantities when integrated (ii) the approach outlined above, while being familiar and having its basis in Maxwell's equations, is nevertheless heuristic. These lead us to ask whether the usual description, centred upon $\mathbf{g}$ and $\mathbf{j}$, is, in fact, the most natural choice. Here, we recognize that quantities such as linear and angular momenta are important, principally, because they are conserved and recall that conservation laws are themselves reflections of symmetries, an idea embodied by Noether's theorem [19, 20]. Hence, a fundamental rather than heuristic approach consists of first identifying appropriate symmetries of Maxwell's equations and subsequently applying Noether's theorem to obtain associated conservation laws, whatever the forms of the latter turn out to be. We adopt this approach in what follows and in doing so, do not arrive at the usual description. Rather, an equally acceptable one in which the relationship between linear and angular momenta is nevertheless more subtle than that embodied by $\mathbf{j}=\mathbf{r} \times \mathbf{g}$ and in which orbital and spin contributions emerge separately and with transparent forms.
The remainder of the paper can be summarized as follows and is depicted in figure 1 . In section 2 , we introduce necessary formalism. In section 3 , we recapitulate Noether's theorem. In section 4, we consider translational symmetry in space, which is traditionally associated with the conservation of linear momentum. We obtain an unfamiliar but acceptable continuity equation that contains information absent from the usual one centred upon g: its azimuthal component is a continuity equation for the $z$ component of orbital angular momentum, with no spin; a surprising but justifiable result. Noether's theorem thus yields, naturally, the idea that orbital angular momentum is an entity unto itself and by extension that the separation of angular momentum into orbital and spin contributions is meaningful. In section 5, we confirm the separation through explicit consideration of the appropriate rotational symmetries. Rotating the spatial distribution of the light without rotating the orientations of the field vectors, we obtain once more a continuity equation for orbital angular momentum. Rotating the orientations of the field vectors without rotating the spatial distribution of the light, we obtain a transparent continuity equation for spin. Combining these yields a complete (geometric) rotation, of course, from which we obtain a continuity equation for the complete (orbital and spin) angular momentum, with separately apparent orbital and spin contributions. In section 6, we reflect upon our findings and identify possible directions for future research.

\section{Formalism}

In what follows, we continue to work in a flat spacetime described primarily by a right-handed Cartesian coordinate system with time $t$ and spatial coordinates $x, y$ and $z$. Where appropriate, we express our results using the language of tensor calculus [21], however, which allows us to consider them also in a related cylindrical coordinate system with time $t$ and spatial coordinates $\rho, \phi$ and $z$ so as to better understand them. We use unprimed indices $\alpha, \beta, \ldots \in\{t, x, y, z\}$ and $a, b, \ldots \in\{x, y, z\}$ for the Cartesian coordinate system and primed indices $\alpha^{\prime}, \beta^{\prime}, \ldots \in\{t, \rho, \phi, z\}$ for the cylindrical coordinate system. Thus, we have the Cartesian position four vector $x^{\alpha}=(t, x, y, z)$ and the cylindrical position four vector $x^{\alpha^{\prime}}=(t, \rho, \phi, z)$. We employ the metric $g_{\alpha \beta}=\operatorname{diag}(-1,1,1,1)$ and the Einstein summation convention throughout [21].

The electric and magnetic fields $\mathbf{E}$ and $\mathbf{B}$ are governed by Maxwell's equations [6]

$$
\begin{aligned}
& \boldsymbol{\nabla} \cdot \mathbf{E}=0, \quad \boldsymbol{\nabla} \times \mathbf{B}=\partial_{t} \mathbf{E}, \\
& \boldsymbol{\nabla} \cdot \mathbf{B}=0, \quad \boldsymbol{\nabla} \times \mathbf{E}=-\partial_{t} \mathbf{B},
\end{aligned}
$$

and can be defined consistently in terms of a scalar potential $\Phi$ and a magnetic vector potential $\mathbf{A}$ as well as a pseudoscalar potential $\Theta$ and an electric pseudovector potential $\mathbf{C}$ as $[22,23]$

$$
\begin{aligned}
& \mathbf{E}=-\nabla \Phi-\partial_{t} \mathbf{A}, \quad \mathbf{B}=\boldsymbol{\nabla} \times \mathbf{A} \\
& \mathbf{B}=-\nabla \Theta-\partial_{t} \mathbf{C}, \quad \mathbf{E}=-\boldsymbol{\nabla} \times \mathbf{C} .
\end{aligned}
$$




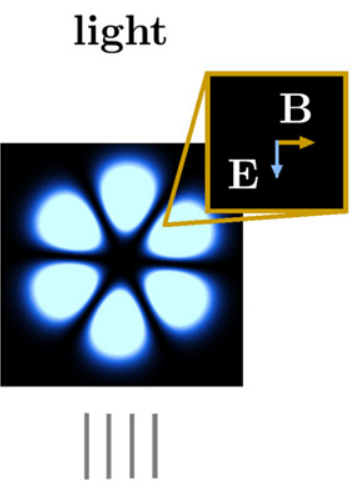

symmetry

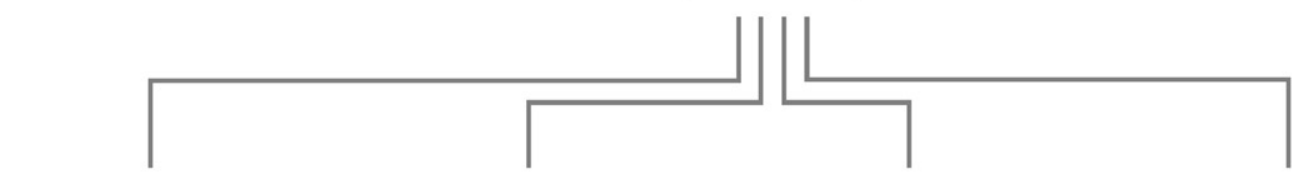

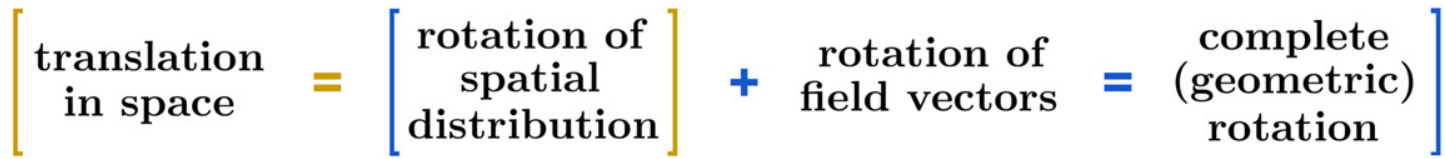

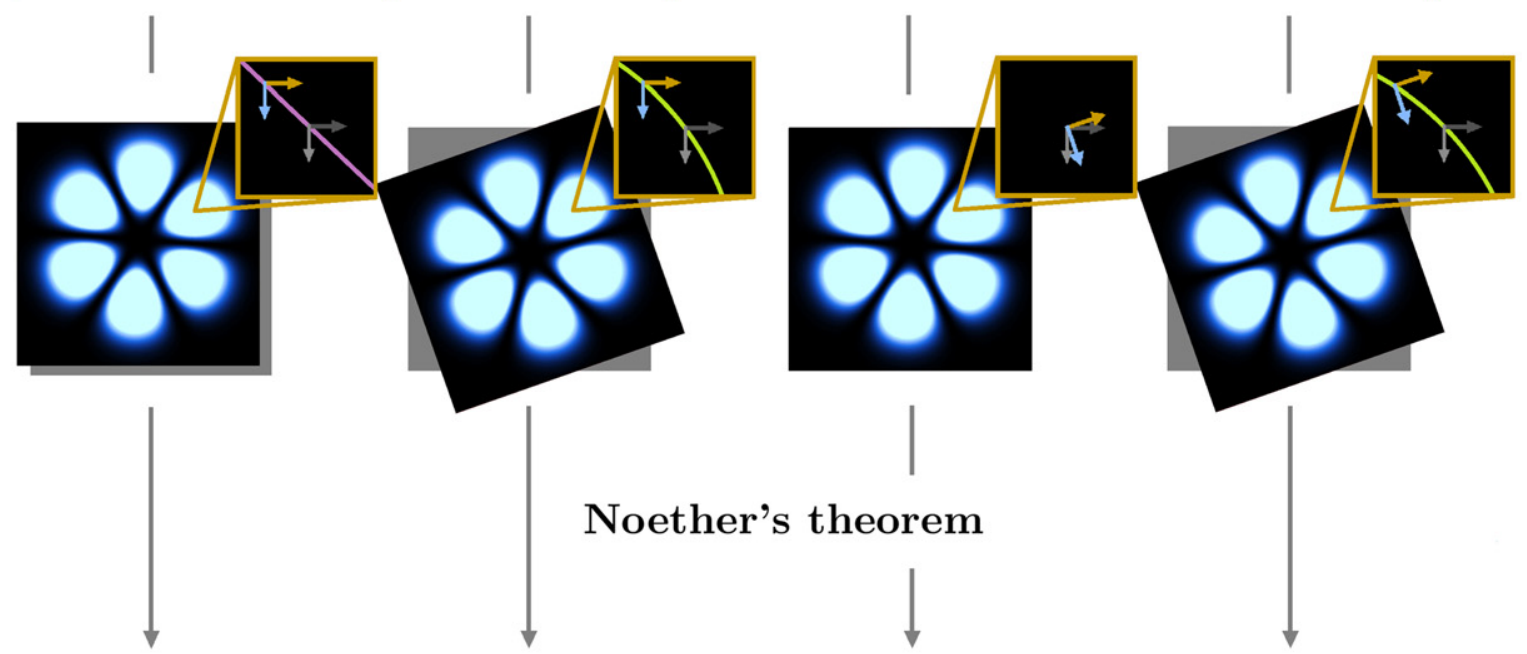

conservation of linear momentum* 8 conservation of orbital angular momentum ${ }^{\dagger}$

$\S 4$

$$
\text { conservation of }
$$$$
\text { orbital }
$$
angular momentum* conservation of spin* conservation of complete (orbital+spin) angular momentum*

$\S 5$

* in Cartesian coordinates

$\dagger$ in cylindrical coordinates

Figure 1. Noether's theorem associates symmetries with conservation laws [19, 20] and affords us a means by which to study the linear and angular momenta of light, as well as the separation of the latter into orbital and spin contributions, from a fundamental rather than heuristic perspective. In the present paper, we consider four subtly related symmetries of Maxwell's equations and their associated conservation laws, as depicted.

These (4) and (5) can be expressed succinctly as

$$
\begin{gathered}
F_{; \beta}^{\alpha \beta}=0, \\
G_{; \beta}^{\alpha \beta}=0
\end{gathered}
$$

and

$$
\begin{aligned}
& F^{\alpha \beta}=A^{\beta ; \alpha}-A^{\alpha ; \beta}, \\
& G^{\alpha \beta}=C^{\beta ; \alpha}-C^{\alpha ; \beta},
\end{aligned}
$$


with $A^{\alpha}=\left(\Phi, A_{x}, A_{y}, A_{z}\right)$ a magnetic potential four vector, $C^{\alpha}=\left(\Theta, C_{x}, C_{y}, C_{z}\right)$ an electric potential four pseudovector, $F^{\alpha \beta}$ the field tensor, $G^{\alpha \beta}$ the dual field pseudotensor and a semicolon indicating covariant differentiation ${ }^{6}$. In matrix form

$$
\begin{aligned}
F^{\alpha \beta} & =\left(\begin{array}{cccc}
0 & E_{x} & E_{y} & E_{z} \\
-E_{x} & 0 & B_{z} & -B_{y} \\
-E_{y} & -B_{z} & 0 & B_{x} \\
-E_{z} & B_{y} & -B_{x} & 0
\end{array}\right), \\
G^{\alpha \beta} & =\left(\begin{array}{cccc}
0 & B_{x} & B_{y} & B_{z} \\
-B_{x} & 0 & -E_{z} & E_{y} \\
-B_{y} & E_{z} & 0 & -E_{x} \\
-B_{z} & -E_{y} & E_{x} & 0
\end{array}\right) .
\end{aligned}
$$

Note that $G^{\alpha \beta}$ is obtained from $F^{\alpha \beta}$ by the transformation $\mathbf{E} \rightarrow \widetilde{\mathbf{E}}=\mathbf{B} \quad$ and $\quad \mathbf{B} \rightarrow \widetilde{\mathbf{B}}=-\mathbf{E} \quad$ or, equivalently, $G^{\alpha \beta}=\epsilon^{\alpha \beta \gamma \delta} F_{\gamma \delta} / 2$, with $\epsilon^{\alpha \beta \gamma \delta}$ the Levi-Civita pseudotensor defined such that $\epsilon^{t x y z}=1$ [21].

\section{Noether's theorem}

In her well known (first) theorem, Noether established that continuous symmetries inherent to the equations of motion governing a system are associated with conservation laws which the system respects $[19,20]$. In electrodynamics in the presence of charge, there exist at least ten continuous symmetries which reflect the homogeneity and isotropy of spacetime. These are invariance under translations in time, translations in the three spatial directions, rotations about the three spatial axes and boosts in the three spatial directions. The conservation laws traditionally associated with these symmetries through Noether's theorem pertain to energy, the three components of linear momentum, the three components of angular momentum and the three components of boost angular momentum [24, 25]. In addition, the symmetry that is gauge invariance is usually associated with the conservation of charge [26]. There exist other symmetries such as invariance under time and parity reversals [6]. Being discrete rather than continuous, such symmetry transformations cannot obviously be brought into an infinitesimal form so as to be investigated using Noether's theorem, however. In the strict absence of charge, as we are considering in the present paper, the situation is richer in that there exists an infinite number of continuous symmetries and associated conservation laws of distinct character [27, 28], in addition to those discussed above. These are seemingly unique to freely propagating light. We shall meet some of them in section 5 .

Various formulations of Noether's theorem are possible. Here, we employ one based upon the electric-magnetic

6 Of course, covariant differentiation happens to reduce in Cartesian coordinates to ordinary partial differentiation [21].
Lagrangian density

$$
\begin{aligned}
\mathcal{L}= & -\frac{1}{8}\left(A_{\beta ; \alpha}-A_{\alpha ; \beta}\right)\left(A^{\beta ; \alpha}-A^{\alpha ; \beta}\right) \\
& -\frac{1}{8}\left(C_{\beta ; \alpha}-C_{\alpha ; \beta}\right)\left(C^{\beta ; \alpha}-C^{\alpha ; \beta}\right)
\end{aligned}
$$

considered in [28, 29]. Applying Hamilton's principle to the action associated with $\mathcal{L}$ while varying the potentials $A^{\alpha}$ and $C^{\alpha}$ independently and subsequently restricting our attention to solutions for which $C^{\beta ; \alpha}-C^{\alpha ; \beta}=\epsilon^{\alpha \beta \gamma \delta}\left(A_{\delta ; \gamma}-A_{\gamma ; \delta}\right) / 2$, we obtain the complete set of Maxwell equations (6) [28]. Imagine now actively transforming $A^{\alpha}$ and $C^{\alpha}$ as

$$
\begin{aligned}
& A^{\alpha} \rightarrow \widetilde{A}^{\alpha}=A^{\alpha}+\delta A^{\alpha}, \\
& C^{\alpha} \rightarrow \widetilde{C}^{\alpha}=C^{\alpha}+\delta C^{\alpha},
\end{aligned}
$$

with the infinitesimal four vector $\delta A^{\alpha}$ and infinitesimal four pseudovector $\delta C^{\alpha}$ satisfying

$$
\delta C^{\beta ; \alpha}-\delta C^{\alpha ; \beta}=\frac{1}{2} \epsilon^{\alpha \beta \gamma \delta}\left(\delta A_{\delta ; \gamma}-\delta A_{\gamma ; \delta}\right) .
$$

This (10) produces a corresponding transformation

$$
\begin{aligned}
& F^{\alpha \beta} \rightarrow \widetilde{F}^{\alpha \beta}=F^{\alpha \beta}+\delta F^{\alpha \beta} \\
& G^{\alpha \beta} \rightarrow \widetilde{G}^{\alpha \beta}=G^{\alpha \beta}+\delta G^{\alpha \beta}
\end{aligned}
$$

of the field and is a symmetry, as the transformed field also satisfies Maxwell's equations:

$$
\begin{aligned}
& \widetilde{F}_{; \beta}^{\alpha \beta}=0, \\
& \widetilde{G}_{; \beta}^{\alpha \beta}=0 .
\end{aligned}
$$

It can be shown using elementary calculus that (10) produces a change $\delta \mathcal{L}$ in $\mathcal{L}$ of the form

$$
\begin{aligned}
\delta \mathcal{L} & =\frac{\partial \mathcal{L}}{\partial A_{\alpha ; \beta}} \delta A_{\alpha ; \beta}+\frac{\partial \mathcal{L}}{\partial C_{\alpha ; \beta}} \delta C_{\alpha ; \beta} \\
& =\left(\frac{\partial \mathcal{L}}{\partial A_{\alpha ; \beta}} \delta A_{\alpha}+\frac{\partial \mathcal{L}}{\partial C_{\alpha ; \beta}} \delta C_{\alpha}\right)_{; \beta} \\
& =\left[\frac{1}{2}\left(F^{\alpha \beta} \delta A_{\alpha}+G^{\alpha \beta} \delta C_{\alpha}\right)\right]_{; \beta},
\end{aligned}
$$

which vanishes because our transformation corresponds to a symmetry. Hence, we are led to the continuity equation

$$
\left[\frac{1}{2}\left(F^{\alpha \beta} \delta A_{\alpha}+G^{\alpha \beta} \delta C_{\alpha}\right)\right]_{; \beta}=0,
$$

which is the form of Noether's theorem that we shall employ in what follows.

For a given symmetry, defined by the transformation (12) of the field, there remains freedom regarding the choice of the underlying transformation (10) of the potentials. Imposition now of the conditions $A^{t}=C^{t}=\boldsymbol{\nabla} \cdot \mathbf{A}=\boldsymbol{\nabla} \cdot \mathbf{C}=0$ so that $\mathbf{A}=\mathbf{A}^{\perp}$ and $\mathbf{C}=\mathbf{C}^{\perp}$, together with the choice of $\delta A^{\alpha}$ and $\delta C^{\alpha}$ such that there is no gauge transformation present in (10), allows us, however, to extract from Noether's theorem (15) a manifestly gauge-invariant continuity equation that embodies the very core of the symmetry. This procedure is to be understood in what follows. To be clear then, $\mathbf{A}$ and $\mathbf{C}$ are to 
be interpreted from here onwards as the gauge-invariant parts $\mathbf{A}^{\perp}$ and $\mathbf{C}^{\perp}$ of the potentials and our final results, given emphatically in terms of $\mathbf{A}^{\perp}$ and $\mathbf{C}^{\perp}$, are independent of gauge.

It is essential, for what follows, to appreciate that neither the locally conserved quantities associated with any given symmetry, nor the fluxes of these, are unique. Poynting's vector, for example, is not the unique momentum density associated with translational symmetry, but rather a convenient choice. We note, moreover, that this freedom in choosing local densities is a general phenomenon, a further example of which is the electromagnetic force density acting on a dielectric with polarisation field $\mathbf{P}$. There are two rival forms for this force [30]:

$$
\begin{aligned}
& \mathbf{f}^{c}=-(\boldsymbol{\nabla} \cdot \mathbf{P}) \mathbf{E}+\partial_{t} \mathbf{P} \times \mathbf{B}, \\
& \mathbf{f}^{d}=(\mathbf{P} \cdot \boldsymbol{\nabla}) \mathbf{E}+\partial_{t} \mathbf{P} \times \mathbf{B} .
\end{aligned}
$$

The former arises from treating the medium as a collection of bound charges and the latter from considering it to be formed from point dipoles. Either form may be used as the total force calculated from either is the same [30]. The difference is simply an illustration of the freedom in choosing the densities of mechanical properties for the electromagnetic field.

\section{Translations and the conservation of linear momentum and orbital angular momentum}

Let us begin now as depicted in the left-most branch of figure 1 by considering an infinitesimal translation of the light in spacetime. We take

$$
F^{\alpha \beta} \rightarrow \widetilde{F}^{\alpha \beta}=F^{\alpha \beta}-K^{\gamma} F_{; \gamma}^{\alpha \beta}
$$

with $K^{\alpha}=\left(\mathcal{K}, K_{x}, K_{y}, K_{z}\right)$ a four vector and the constants $\mathcal{K}$, $K_{x}, K_{y}$ and $K_{z}$ infinitesimal ${ }^{7}$. It follows immediately ${ }^{8}$ from Noether's theorem (15) that

$$
K^{\alpha} T_{\alpha ; \beta}^{\beta}=0
$$

with $^{9}$

$$
T_{\alpha}^{\beta}=\frac{1}{2}\left(F^{\beta \gamma} A_{\gamma_{; \alpha}}+G^{\beta \gamma} C_{\gamma_{; \alpha}}\right) .
$$

As $\mathcal{K}, K_{x}, K_{y}$ and $K_{z}$ are independent and essentially arbitrary, we deduce from (18) that

$$
T_{\alpha ; \beta}^{\beta}=0,
$$

which may be identified as a continuity equation for energy $(\alpha=t)$ and the $x, y, z$ components of linear momentum ( $\alpha=x, y, z$ ) owing to the nature of the symmetry transformation (17), with $T_{\alpha}{ }^{\beta}$ an energy-momentum tensor accordingly.

7 Note that $K^{\alpha} ; \beta=0$.

${ }^{8}$ With $\delta A_{\alpha}=-K^{\beta} A_{\alpha ; \beta}$ and $\delta C_{\alpha}=-K^{\beta} C_{\alpha ; \beta}$.

9 The reader may recognize that $T_{\alpha}{ }^{\beta}$ resembles the gauge-invariant piece of the 'canonical' energy-momentum tensor obtainable from the standard rather than electric-magnetic Lagrangian density while being distinct from it: see $[6,21,31]$, for example. The precise form taken by $T_{\alpha}{ }^{\beta}$ evidently differs for different choices of Lagrangian density; another manifestation of nonuniqueness [32]. The ideas introduced in the present paper hold regardless of such choices, however.
Somewhat suprisingly, $T_{\alpha}{ }^{\beta}$ is not the form

$$
W_{\alpha}^{\beta}=\frac{1}{2}\left(F^{\beta \gamma} F_{\alpha \gamma}+G^{\beta \gamma} G_{\alpha \gamma}\right)\left(W_{\alpha}^{\beta} ; \beta=0\right)
$$

traditionally recognized for the energy-momentum tensor. In particular, the components

$$
T_{a}^{t}=g_{a}+\partial_{b} \frac{1}{2}\left(A_{a} E_{b}+C_{a} B_{b}\right)
$$

differ from those $g_{a}$ of Poynting's vector $\mathbf{g}$ by a total spatial divergence, as indicated. At this stage, $T_{\alpha}{ }^{\beta}$ is usually rejected: in addition to its unfamiliarity, $T_{\alpha}{ }^{\beta}$ is neither symmetric $\left(T^{\alpha \beta} \neq T^{\beta \alpha}\right)$ nor traceless $\left(T_{\alpha}^{\alpha} \neq 0\right)$ and it is claimed that the absence of these characteristics jeopardises the conservation of angular momentum, boost angular momentum and boost helicity [6, 31]. Various ad hoc procedures have been conceived of to yield the traditionally recognized energymomentum tensor $W_{\alpha}{ }^{\beta}$ rather than $T_{\alpha}{ }^{\beta}$ when considering translational symmetry and applying Noether's theorem, the simplest of which is to add $W_{\alpha}{ }^{\beta}-T_{\alpha}{ }^{\beta}$ to $T_{\alpha}{ }^{\beta}[6,24,31-33]$.

We recognize in spite of the above that the $T_{a^{t}}$ are acceptable as the components of a linear momentum density: although unfamiliar, they yield the components of the total linear momentum when integrated over all space just as the $g_{a}$ do and neither is to be preferred in this context. Moreover, the claim $[6,31]$ that the characteristics of an energy-momentum tensor influence the conservation of angular momentum, boost angular momentum and boost helicity is unjustified, as we shall demonstrate in section 5. Rather than attempting to fix Noether's theorem, let us therefore attempt instead to understand the result that it has yielded. To this end, we now take advantage of the tensorial character of (20) and examine (20) in the cylindrical coordinate system $x^{\alpha^{\prime}}$. Considering the azimuthal component and making use of the tensor transformation law relating $x^{\alpha^{\prime}}$ to $x^{\alpha}$, we find that

$$
\begin{aligned}
T_{\phi}{ }^{\alpha^{\prime} \alpha^{\prime}} & =\frac{\partial x^{\alpha}}{\partial x^{\phi}} T_{\alpha}^{\beta} ; \beta \\
& =\partial_{t} l_{z}+\partial_{a} o_{z a} \\
& =0
\end{aligned}
$$

which is a continuity equation not for linear momentum but rather for the $z$ component of orbital angular momentum, with

$$
\mathbf{l}=\frac{1}{2}\left[E_{a}(\mathbf{r} \times \nabla) A_{a}+B_{a}(\mathbf{r} \times \nabla) C_{a}\right]
$$

an orbital angular momentum density [18] and

$$
\begin{aligned}
o_{a b}= & \frac{1}{2}\left[\epsilon_{a c d} \epsilon_{b e f}\left(E_{e} r_{c} \partial_{d} C_{f}-B_{e} r_{c} \partial_{d} A_{f}\right)\right. \\
& \left.+A_{a} B_{b}-C_{a} E_{b}\right]
\end{aligned}
$$

the components of an orbital angular momentum flux density [34], $\epsilon_{a b c}$ being the Levi-Civita rotational pseudotensor defined such that $\epsilon_{x y z}=1$ [21]. We can understand this in turn by noting that

$$
\begin{aligned}
& K^{t}=\mathcal{K}, K^{\rho}=\hat{\boldsymbol{\rho}} \cdot \mathbf{K}, K^{\phi}=\hat{\boldsymbol{\phi}} \cdot \mathbf{K} / \rho, \\
& K^{z}=\hat{\mathbf{z}} \cdot \mathbf{K},
\end{aligned}
$$




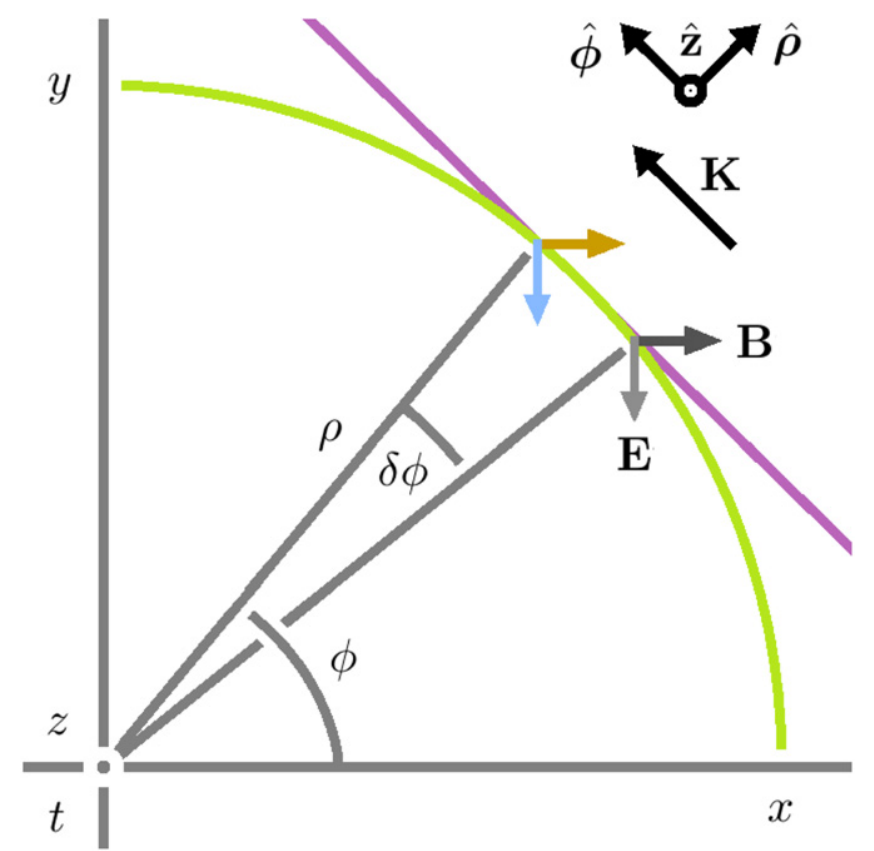

Figure 2. For any given $t, \rho, \phi$ and $z$, an infinitesimal translation of the light in space through $\mathbf{K}=\rho \delta \phi \hat{\boldsymbol{\phi}}$ (parallel to the purple line) coincides with an infinitesimal rotation of the spatial distribution of the light about the $z$ axis through $\delta \phi$ (indicated by the light green curve), with no rotation of the orientations of the field vectors.

with $\hat{\boldsymbol{\rho}}, \hat{\boldsymbol{\phi}}$ and $\hat{\mathbf{z}}$ unit vectors in the directions of increasing $\rho, \phi$ and $z$. The extraction of (23) from (18) thus follows from an effective choice of $\mathcal{K}=0$ and $\mathbf{K}=\rho \delta \phi \hat{\boldsymbol{\phi}}$ at the given $t, \rho, \phi$ and $z$, with $\delta \phi$ an infinitesimal angle. As depicted in figure 2, the translation so defined coincides with a rotation of the spatial distribution of the light about the $z$ axis through $\delta \phi$, with no rotation of the orientations of the field vectors. Hence the emergence of a continuity equation (23) for the $z$ component of orbital angular momentum, with no spin. Thus, we see now that it is unreasonable to expect Noether's theorem to yield a continuity equation centred upon $\mathbf{g}$ in connection with translations in space: as the azimuthal component $j_{z}$ of $\mathbf{g}$ describes orbital angular momentum and spin, albeit obscurely, this would be tantamount to obtaining information about spin without rotating the orientations of the field vectors!

In summary, the continuity equation yielded by Noether's theorem in connection with translations in space, although unfamiliar, is nevertheless acceptable and in fact contains information absent from the usual one centred upon g: Noether's theorem yields, naturally, the idea that orbital angular momentum is an entity unto itself and by extension that the separation of angular momentum into orbital and spin contributions is meaningful. This is obscured by the use of Cartesian coordinates but emerges in cylindrical coordinates.

\section{Rotations and the conservation of orbital angular momentum and spin}

We now confirm the separation of angular momentum into orbital and spin contributions through explicit consideration of the appropriate rotational symmetries in the Cartesian coordinate system $x^{\alpha}$.

First, we proceed as depicted in the middle-left branch of figure 1 and consider an infinitesimal rotation of the spatial distribution of the light without rotating the orientations of the field vectors ${ }^{10}$, taking [18]

$$
\begin{aligned}
& \mathbf{E} \rightarrow \widetilde{\mathbf{E}}=\mathbf{E}-[\boldsymbol{\theta} \cdot(\mathbf{r} \times \nabla) \mathbf{E}]^{\perp}, \\
& \mathbf{B} \rightarrow \widetilde{\mathbf{B}}=\mathbf{B}-[\boldsymbol{\theta} \cdot(\mathbf{r} \times \nabla) \mathbf{B}]^{\perp},
\end{aligned}
$$

with the components of $\boldsymbol{\theta}$ infinitesimal and constant. Employing Noether's theorem (15) and making some simple manipulations ${ }^{11}$ to the result that follows, we associate (27) with

$$
\partial_{t} l_{a}+\partial_{b} o_{a b}=0,
$$

which is a continuity equation for orbital angular momentum [34], the $z$ component of which coincides with (23). When integrated over all space, (28) yields the global conservation of orbital angular momentum obtained in $[15,16,28]$.

Second, we proceed as depicted in the middle-right branch of figure 1 and consider an infinitesimal rotation of the orientations of the field vectors without rotating the spatial distribution of the light, taking $[15,16,18]$

$$
\begin{aligned}
& \mathbf{E} \rightarrow \widetilde{\mathbf{E}}=\mathbf{E}+(\boldsymbol{\theta} \times \mathbf{E})^{\perp}, \\
& \mathbf{B} \rightarrow \widetilde{\mathbf{B}}=\mathbf{B}+(\boldsymbol{\theta} \times \mathbf{B})^{\perp} .
\end{aligned}
$$

Again employing (15) and making some simple manipulations to the result that follows, we associate (29) with

$$
\partial_{t} s_{a}+\partial_{b} n_{a b}=0,
$$

which is a transparent continuity equation for spin [27], with

$$
\mathbf{s}=\frac{1}{2}(\mathbf{E} \times \mathbf{A}+\mathbf{B} \times \mathbf{C})
$$

a spin density and

$$
\begin{aligned}
n_{a b}= & \frac{1}{2}\left[\delta_{a b}(\mathbf{A} \cdot \mathbf{B}-\mathbf{C} \cdot \mathbf{E})\right. \\
& \left.-A_{a} B_{b}-A_{b} B_{a}+C_{a} E_{b}+C_{b} E_{a}\right]
\end{aligned}
$$

the components of a spin flux density. When integrated over all space, (30) yields the global conservation of spin obtained in $[15,16,27,28]$.

Finally, we proceed as depicted in the right-most branch of figure 1 by considering an infinitesimal complete (geometric) rotation of the light, taking [18]

$$
\begin{aligned}
& \mathbf{E} \rightarrow \widetilde{\mathbf{E}}=\mathbf{E}+\boldsymbol{\theta} \times \mathbf{E}-\boldsymbol{\theta} \cdot(\mathbf{r} \times \nabla) \mathbf{E}, \\
& \mathbf{B} \rightarrow \widetilde{\mathbf{B}}=\mathbf{B}+\boldsymbol{\theta} \times \mathbf{B}-\boldsymbol{\theta} \cdot(\mathbf{r} \times \nabla) \mathbf{B},
\end{aligned}
$$

which is of course the combination of (27) and (29). Employing (15) once more, we associate (33) with

$$
\partial_{t}\left(l_{a}+s_{a}\right)+\partial_{b}\left(o_{a b}+n_{a b}\right)=0,
$$

10 Strictly speaking, we consider the closest permissible approximation to this rotation [18].

11 The need to perform these is attributable to the solenoidal character of $\mathbf{E}$ and B. Superficially, the $\perp$ symbol in (27). 
which is a continuity equation for the complete (orbital and spin) angular momentum, centred not upon the usual angular momentum density $\mathbf{j}$ but rather an equally acceptable angular momentum density $\mathbf{l}+\mathbf{s}$ in which orbital and spin contributions are nevertheless separately apparent. For a single circularly polarized plane wave of angular frequency $\omega$ propagating in the $+z$ direction, we find that

$$
\begin{gathered}
l_{z}=0, \\
\frac{s_{z}}{\overline{T^{t} t}}=\frac{ \pm \hbar}{\hbar \omega},
\end{gathered}
$$

with an overbar indicating a cycle average and where the plus and minus signs refer to left- and right-handed circular polarisation in the optics convention. These seemingly natural $[5,12]$ results $(35)$ suggest a spin of $\pm \hbar \hat{\mathbf{z}}$ 'per photon' and should be compared with that seen in (3). For a single linearly polarized Bessel beam of angular frequency $\omega$ and winding number $\ell$ propagating in the $+z$ direction, we find that

$$
\begin{aligned}
\frac{\bar{l}_{z}}{\overline{T^{t} t}} & =\frac{\hbar \ell}{\hbar \omega}, \\
s_{z} & =0,
\end{aligned}
$$

suggesting an orbital angular momentum of $\hbar \ell \hat{\mathbf{z}}$ 'per photon', which again seems natural [4].

We did not consider $T_{\alpha}{ }^{\beta}$ in arriving at (34). Evidently then, the conservation of angular momentum does not rely upon the characteristics of an energy-momentum tensor. Analogous observations hold for the conservation of boost angular momentum and boost helicity. The fact that $\mathbf{j}$ is the cross product of position $\mathbf{r}$ with Poynting's vector $\mathbf{g}$ is not indicative of a general relationship between linear and angular momentum: clearly, $\mathbf{l}+\mathbf{s}$ is not the cross product of $\mathbf{r}$ with the $T_{a}^{t}$, as $l_{x}+s_{x} \neq y T_{z}^{t}-z T_{y}^{t}$ and similarly for the $y$ and $z$ components. This role is reserved instead for orbital angular momentum, with no spin, as we saw in section 4 .

Note that the separate orbital and spin contributions obtained as above do not correspond with the so-called 'orbital' and 'spin' tensors identified heuristically elsewhere $[32,33]$ : the latter each have suggestive forms but nevertheless are not separately associated with symmetry transformations and do not separately obey continuity equations. Let us emphasize here once more that our final results, expressed emphatically in terms of $\mathbf{A}=\mathbf{A}^{\perp}$ and $\mathbf{C}=\mathbf{C}^{\perp}$, embody the very cores of our symmetries and are independent of gauge.

\section{Discussion}

We have approached the linear and angular momentum of light, as well as the separation of the latter into orbital and spin contributions, from a fundamental rather than heuristic perspective by first identifying symmetries of Maxwell's equations and subsequently applying Noether's theorem to obtain associated conservation laws. We did not arrive at the usual description, centred upon the identification of Poynting's vector $\mathbf{g}$ as a linear momentum density and its cross product $\mathbf{j}$ with position $\mathbf{r}$ as an angular momentum density.
Rather, an equally acceptable and perhaps preferable one in which the relationship between linear and angular momentum is nevertheless more subtle than that embodied by $\mathbf{j}=\mathbf{r} \times \mathbf{g}$ and in which orbital and spin contributions emerge separately and with transparent forms.

That a given symmetry can be associated with different conservation laws through the use of different coordinate systems appears worthy of further study. It would also be interesting to see how our present findings generalize to the consideration of curved spacetimes, particularly as the energy momentum tensor $W_{\alpha}{ }^{\beta}$ is given a seemingly privileged status by Einstein's theory of gravitation [21, 32, 35]. We shall return to these ideas elsewhere.

\section{Acknowledgments}

This work was supported by EPSRC grants EP/101245/1 and EP/M004694/1 and The Carnegie Trust for the Universities of Scotland.

\section{References}

[1] Allen L, Barnett S M and Padgett M J 2003 Optical Angular Momentum (Bristol: Institute of Physics)

[2] Yao A M and Padgett M J 2011 Orbital angular momentum: origins, behavior and applications Adv. Opt. Photonics 3 161-204

[3] Andrews D L and Babiker M 2013 The Angular Momentum of Light (Cambridge: Cambridge University Press)

[4] Allen L, Beifersbergen M W, Spreeuw R J C and Woerdman J P 1992 Orbital angular momentum of light and the transformation of Laguerre-Gaussian laser modes Phys. Rev. A 45 8185-9

[5] Poynting J H 1909 The wave motion of a revolving shaft, and a suggestion as to the angular momentum in a beam of circularly polarised light Proc. R. Soc. A 82 560-7

[6] Jackson J D 1999 Classical Electrodynamics (New York: Wiley)

[7] Poynting J H 1884 On the transfer of energy in the electromagnetic field Phil. Trans. R. Soc. Lond. $175343-61$

[8] Darwin C G 1932 Notes on the theory of radiation Proc. $R$. Soc. A 136 36-52

[9] Humblet J 1943 Sur le moment d'impulsion d'une onde électromagnétique Physica 10 585-603

[10] Cohen Tannoudji C, Dupont-Roc J and Grynberg G 1989 Photons and Atoms (New York: Wiley)

[11] Allen L and Padgett M J 2002 Response to Question \#79. Does a plane wave carry spin angular momentum? Am. J. Phys. 70 567-8

[12] Beth R A 1936 Mechanical detection and measurement of the angular momentum of light Phys. Rev. 50 115-25

[13] Simmons W and Guttmann M J 1970 States, Waves and Photons (Reading: Addison-Wesley)

[14] Speirits F C and Barnett S M 2013 Do waves carrying orbital angular momentum possess azimuthal linear momentum? Phys. Rev. Lett. 111103602

[15] van Enk S J and Nienhuis G 1994 Spin and orbital angular momentum of photons Europhys. Lett. 25 497-501

[16] van Enk S J and Nienhuis G 1994 Commutation rules and eigenvalues of spin and orbital angular momentum of radiation fields J. Mod. Opt. 41 963-77 
[17] Zambrini R and Barnett S M 2004 Local transfer of optical angular momentum of matter J. Mod. Opt. 52 1045-52

[18] Barnett S M 2010 Rotation of electromagnetic fields and the nature of optical angular momentum J. Mod. Opt. 57 1339-43

[19] Noether E 1918 Invariante variationsprobleme Nachr. D. König. Gesellsch. D. Wiss. Zu Göttingen, Math-phys. Klasse 2 235-57

[20] Neuenschwander D E 2011 Emmy Noether's Wonderful Theorem (Baltimore: John Hopkins University Press)

[21] Landau L D and Lifshitz E M 1975 The Classical Theory of Fields (Oxford: Butterworth-Heinemann)

[22] Bateman H 1915 The Mathematical Analysis of Electrical and Optical Wave-Motion on the Basis of Maxwell's Equations (Cambridge: Cambridge University Press)

[23] Cameron R P 2014 On the 'second potential' in electrodynamics J. Opt. 16015708

[24] Bessel-Hagen E 1921 Über die erhaltungssätze der elektrodynamik Math Ann. 84 258-76

[25] Barnett S M 2011 On the six components of optical angular momentum J. Opt. 13064010

[26] Weyl H 1918 Gravitat und Elektrizität Sitzungsber. Preuss. Akad. Wiss. 26 465-80
[27] Cameron R P, Barnett S M and Yao A M 2012 Optical helicity, optical spin and related quantities in electromagnetic theory New. J. Phys. 14053050

[28] Cameron R P and Barnett S M 2012 Electric-magnetic symmetry and Noether's theorem New J. Phys. 14123019

[29] Bliokh K Y, Bekshaev A Y and Nori F 2012 Dual electromagnetism: helicity, spin, momentum and angular momentum New J. Phys. 15033026

[30] Barnett S M and Loudon R 2006 On the electromagnetic force on a dielectric medium J. Phys. B: At. Mol. Opt. Phys. 39 S671

[31] Goldstein H 1985 Classical Mechanics (Singapore: AddisonWesley)

[32] Belinfante F J 1940 On the current and the density of the electric charge, the energy, the linear momentum and the angular momentum of arbitrary fields Physica 7 449-74

[33] Belinfante F J 1939 On the spin angular momentum of mesons Physica 6 887-98

[34] Bliokh K, Dressel J and Nori F 2014 Conservation of the spin and orbital angular momenta in electromagnetism New J. Phys. 16093037

[35] Barnett S M 2014 Maxwellian theory of gravitational waves and their mechanical properties New. J. Phys. 16023027 